OPEN ACCESS

Edited by:

Fabian Cieplik,

University Medical Center

Regensburg, Germany

Reviewed by:

Divakar Sharma,

Indian Institute of Technology Delhi,

India

Haruyoshi Tomita,

Gunma University, Japan

*Correspondence:

Xiaohui Huang

math2088@163.com

Jiabin Li

lijiabin@ahmu.edu.cn

Specialty section: This article was submitted to Antimicrobials, Resistance

and Chemotherapy,

a section of the journal

Frontiers in Microbiology

Received: 14 September 2020 Accepted: 30 December 2020 Published: 09 February 2021

Citation:

Jiang L, Xie N, Chen M, LiU Y,

Wang S, Mao J, Li J and Huang $X$ (2021) Synergistic Combination

of Linezolid and Fosfomycin Closing

Each Other's Mutant Selection

Window to Prevent Enterococcal

Resistance.

Front. Microbiol. 11:605962.

doi: 10.3389/fmicb.2020.605962

\section{Synergistic Combination of Linezolid and Fosfomycin Closing Each Other's Mutant Selection Window to Prevent Enterococcal Resistance}

\author{
Lifang Jiang 1,2, Na Xie ${ }^{1,2}$, Mingtao Chen 1,2, Yanyan Liü3, Shuaishuai Wang ${ }^{1,2}$, Jun Mao ${ }^{1,2}$, \\ Jiabin $\mathrm{Li}^{3 *}$ and Xiaohui Huang ${ }^{1,2 *}$ \\ ' Department of Basic and Clinical Pharmacology, School of Pharmacy, Anhui Medical University, Hefei, China, ${ }^{2}$ Anhui \\ Province Key Laboratory of Major Autoimmune Diseases, School of Pharmacy, Anhui Institute of Innovative Drugs, Anhui \\ Medical University, Hefei, China, ${ }^{3}$ Department of Infectious Diseases, The First Affiliated Hospital of Anhui Medical University, \\ Hefei, China
}

Enterococci, the main pathogens associated with nosocomial infections, are resistant to many common antibacterial drugs including $\beta$-lactams, aminoglycosides, etc. Combination therapy is considered an effective way to prevent bacterial resistance. Preliminary studies in our group have shown that linezolid combined with fosfomycin has synergistic or additive antibacterial activity against enterococci, while the ability of the combination to prevent resistance remains unknown. In this study, we determined mutant prevention concentration (MPC) and mutant selection window (MSW) of linezolid, fosfomycin alone and in combination including different proportions for five clinical isolates of Enterococcus and characterized the resistance mechanism for resistant mutants. The results indicated that different proportions of linezolid combined with fosfomycin had presented different MPCs and MSWs. Compared with linezolid or fosfomycin alone, the combination can restrict the enrichment of resistant mutants at a lower concentration. A rough positive correlation between the selection index (SI) of the two agents in combination and the fractional inhibitory concentration index $(\mathrm{FICl})$ of the combination displayed that the smaller $\mathrm{FICl}$ of linezolid and fosfomycin, the more probable their MSWs were to close each other. Mutations in ribosomal proteins ( $L 3$ and L4) were the mechanisms for linezolid resistant mutants. Among the fosfomycin-resistant mutants, only two strains have detected the MurA gene mutation related to fosfomycin resistance. In conclusion, the synergistic combination of linezolid and fosfomycin closing each other's MSW could effectively suppress the selection of enterococcus resistant mutants, suggesting that the combination may be an alternative for preventing enterococcal resistance. In this study, the resistance mechanism of fosfomycin remains to be further studied.

Keywords: MPC, MSW, linezolid, fosfomycin, Enterococcus 


\section{INTRODUCTION}

Enterococci are one of prominent causes of hospital acquired infection, especially in urinary tract, soft tissue, and deviceassociated infections (Fiore et al., 2019; García-Solache and Rice, 2019). Enterococcus faecalis and Enterococcus faecium are the main pathogenic bacteria of enterococcal infections (Gilmore et al., 2013). Both the two species shown intrinsic resistance to common antibiotics historically used as front-line agents, making enterococcal infection to be a serious threat to public health (Mercuro et al., 2018; Torres et al., 2018; Haghi et al., 2019). Combination therapy is recommended as an effective method to combat bacterial resistance (Tyers and Wright, 2019). Clinical studies also shown that patients treated with antibacterial combination therapy can obtain good clinical effect and lower mortality rates (Falagas et al., 2014; Ni et al., 2015). Currently, a variety of synergistic and effective combinations against enterococcal infections have been reported (Leone et al., 2016; Mercuro et al., 2018). However, most studies were aimed at exploring the antibacterial activity of the combination in vitro or in vivo (Mercuro et al., 2018). There are few researches about combinations that can effectively prevent enterococcal resistance.

One approach to predict bacterial resistance to antimicrobials in vitro was determination of the mutant prevention concentration (MPC) and mutant selection window (MSW). The MSW comprises a specific drug concentration range in which mutant strains with reduced susceptibility can be selected (Drlica and Zhao, 2007; Blondeau, 2009). It has been confirmed in many antibiotics such as fluoroquinolones (Strukova et al., 2016), oxazolidinones (Alieva et al., 2018), aminoglycosides ( $\mathrm{Ni}$ et al., 2016). MPC is defined as the lowest concentration that blocks the emergence of first-step resistant mutants in a large susceptible population, usually more than $10^{10}$ colony forming unit (CFU)/mL bacteria (Drlica, 2003). Maintaining the drug concentration above MPC can effectively restrict the selection resistant subpopulations (Zinner et al., 2008; Alieva et al., 2018). In practice, high exposure was related to higher incidence of side effects. Fortunately, combination therapy at a low concentration could prevent the selection of resistant mutants by narrowing or closing the MSW (Díez-Aguilar et al., 2015; Ni et al., 2016). Theoretically, the more likely the combination is to shut off each other's MSW at the same time, the stronger its ability to prevent bacterial resistance. Therefore, finding a combination that can close each other's MSW at the same time to achieve therapeutic effect with lower dose was the key to preventing enterococcal resistance.

Linezolid is used as the first-line drug for the treatment of severe gram-positive infections, instead of vancomycin (Zahedi Bialvaei et al., 2017). Although, some studies have shown that the frequency of spontaneous resistance to linezolid was low in enterococci (L. Drago et al., 2008). Unfortunately, in recent years, the increasing number of linezolid resistant enterococci had been reported worldwide (Sassi et al., 2019; Zou and Xia, 2020). Even, some studies revealed that prolonged linezolid therapy was regarded as a risk factor for the obtaining of linezolid resistant E. faecium clinical isolates (Smith et al., 2018). And high linezolid consumption facilitates the development of linezolid resistant
E. faecalis (Bai et al., 2019). In addition, with the increase in exposure and treatment time, linezolid may cause higher rates of adverse reactions such as thrombocytopenia and neuropathy (Bayram et al., 2017; Tsuji et al., 2017; Lee and Caffrey, 2018). Considering the limitations of linezolid monotherapy, the use of a combination strategy may be a good approach. Fosfomycin acts on bacterial cell walls and shows good antibacterial activity against gram-positive and gram-negative bacteria including multi-drug resistant bacteria. Fosfomycin, due to its unique mechanism of action, has a synergistic effect with a variety of antibiotics and is not easy to produce cross-resistance (Falagas et al., 2016). The previous study of our group confirmed that linezolid combined with fosfomycin has an in vitro synergistic effect on Enterococcus and Staphylococcus aureus (Chen et al., 2018; Qi et al., 2019). However, the ability of the combination to prevent enterococcus resistance remains to be studied.

Understanding the mechanism of bacterial resistance is of great significance for guiding the rational application of antibiotics, preventing bacterial drug resistance and effective anti-infection treatment. However, the mechanisms of bacterial resistance are complex. On the one hand, bacteria can become resistant by acquiring either exogenous resistance genes or chromosomal mutations (Durão et al., 2018). On the other hand, the drug-resistant phenotype of bacteria can also be expressed through changes in protein levels (Sharma et al., 2019a,b). Although, the drug resistance mechanism of clinically isolated linezolid-resistant enterococci (Hua et al., 2019; Zou and Xia, 2020) or fosfomycin-resistant enterococci has been reported (Zhang et al., 2019). But, mechanisms responsible for linezolid or fosfomycin resistant mutants selected from the MSW are still lacking. The emergence of first-step mutants during the MPC measurement offers the possibility to explore the mechanisms of resistance in the molecular level.

To support the clinical application of this combination, this study is the first in vitro to evaluate the ability of linezolid combined with fosfomycin in different proportions to prevent enterococcal resistance by determining the MSW of the two agents when used alone or in combination. Similarly, this study conducted a preliminary exploration of the resistance mechanism of linezolid or fosfomycin resistant mutants.

\section{MATERIALS AND METHODS}

\section{Bacterial Isolates}

Number of 43 non-duplicate clinical isolates of Enterococcus were isolated from urine, blood, bile, pus, and excrement between January and October 2018 in the First Affiliated Hospital of Anhui Medical University. Among them, 20 strains of Enterococcus faecium, 23 strains of Enterococcus faecalis. All strains were identified by the automated VITEK-2 system (BioMerieux, Marcy l'Etoile, France). Enterococcus faecalis ATCC 29212 was used as the quality control strain. In addition, these strains were not specifically isolated for this research but were part of the routine hospital laboratory procedure. This study was approved by the First Affiliated Hospital of Anhui Medical University institutional review board. 


\section{Antimicrobial Agents and Medium}

Linezolid and fosfomycin were purchased from the National Institute for Food and Drug Control of China (Beijing, China). Mueller-Hinton broth (MHB, Oxoid, England) was used for culturing bacteria and Mueller-Hinton agar (MHA, Oxoid, England) was used for culturing bacteria, performing agar dilution method and quantifying colony counts.

\section{Determination of Antimicrobial Susceptibility}

The minimum inhibitory concentration (MIC) for the two drugs was determined by agar dilution according to Clinical and Laboratory Standards Institute (Clsi, 2019) guidelines. Briefly, Mueller Hinton agar (MHA; Oxoid, England) plates containing a series of 2-fold concentration increments of each agent were prepared. The agar plates containing fosfomycin needs to add glucose-6-phosphate and makes the final concentration $25 \mathrm{mg} / \mathrm{L}$. Then, $\sim 10^{5}$ colony-forming units (CFU) of bacterial cells were inoculated with an autoclaved replicator and incubated at $37^{\circ} \mathrm{C}$ for $24 \mathrm{~h}$. The MIC was defined as the lowest drug concentration in which no visible colonies grew. Enterococcus faecalis ATCC 29212 was used as the quality control strain in each batch of tests. The experiment was replicated three times.

\section{Checkerboard Assays}

Checkerboard assay was used for the synergy testing. Tests were performed on 96-well plates according to our previous study (Qi et al., 2019), the two drugs were diluted with Mueller-Hinton Broth into a series of concentrations based on the MICs for each tested isolate. In brief, linezolid ranging between 1/64 $\times$ MIC and $2 \times$ MIC was dispensed in each column. Then, fosfomycin supplemented with $25 \mathrm{mg} / \mathrm{L}$ of glucose-6-phosphate ranging from $1 / 64 \times$ MIC to $2 \times$ MIC was added in every row. Then, each well was inoculated with an equal volume of $1 \times 10^{6} \mathrm{CFU} / \mathrm{mL}$ bacterial suspension. Plates were incubated at $37^{\circ} \mathrm{C}$ for $24 \mathrm{~h}$ and visually inspected for turbidity to determine the growth. All the experiments were performed in triplicate.

Synergy was evaluated by the fractional inhibitory concentration index (FICI): FICI $=($ MIC of drug $\mathrm{A}$ in combination/MIC of drug $\mathrm{A}$ alone) + (MIC of drug $\mathrm{B}$ in combination/MIC of drug $\mathrm{B}$ alone). The FICI value was interpreted as follows: FICI $\leq 0.5$, synergy; $0.5<$ FICI $\leq 1$, additivity; $1<$ FICI $\leq 4$, indifference; FICI $>4$, antagonism (Davis et al., 2020).

\section{MIC99\%}

According to the results of checkerboard assay, five isolates (Enterococcus faecium: NO.1, NO.5; Enterococcus faecalis: NO.6, NO.22, NO.43) with different value of FICI were selected for the MSW studies. For the five selected strains, the $\mathrm{MIC}_{99 \%}$ of linezolid and Fosfomycin were determined repeatedly by the agar plate methods reported in previous research (Xu et al., 2018). In short, bacterial suspension was inoculated on agar plates including linear drug concentrations with $20 \%$ per sequential decrease from each MIC, and those plates without drug used for blank controls. The fosfomycin-containing agar plates were required to supplement with glucose-6-phosphate at a final concentration of $25 \mathrm{mg} / \mathrm{L}$. The plates were incubated at $35^{\circ} \mathrm{C}$ for $24 \mathrm{~h}$, and then the colonies growing on different plates were counted. Finally, calculate the inhibition percentage (y) and plot against different antibacterial agent concentrations ( $\mathrm{x}$ ) to gain a regression equation. Accordingly, their $\mathrm{MIC}_{99 \%}$ were, respectively, calculated according to their individual equations.

\section{MPC Alone or in Combination}

Linezolid, fosfomycin, and linezolid-fosfomycin combination MPCs for five isolates (NO.1, NO.5, NO.6, NO.22, and NO.43) were determined according to the method reported in previous studies (Wentao et al., 2018). In brief, bacterial cells were grown overnight in fresh Mueller-Hinton broth (MHB) with violently shaking at $35^{\circ} \mathrm{C}$ and followed by 10 -fold dilution with $\mathrm{MHB}$, incubated at $35^{\circ} \mathrm{C}$ for $6 \mathrm{~h}$. The growth was centrifuged $(4000 \times g$ for $10 \mathrm{~min}$ ) to yield a high-density culture containing cells of $\sim 10^{10} \mathrm{CFU} / \mathrm{ml}$. One hundred microliter culture (approximate $\sim 10^{9}$ cell) was placed onto Mueller-Hinton agar plates with 2fold increasing concentrations. Also, the glucose-6-phosphate at a final concentration of $25 \mathrm{mg} / \mathrm{L}$ need to be added into agar plates containing fosfomycin. Then, the plates were incubated at $35^{\circ} \mathrm{C}$ for $72 \mathrm{~h}$. The preliminary MPC was recorded as the lowest antimicrobial concentration that prevented bacterial growth. Further, the exact MPC was determined by linear antimicrobial concentration with $20 \%$ per sequential decrease from preliminary MPC. Based on the FICI value of the combination, thirteen different composition ratios (Linezolid: Fosfomycin, from 64:1 to 1:64) were designed for the combination MPCs studies. The MPCs of different proportions of linezolid combined with fosfomycin were determined according to above methods and procedures. For each strain, colonies that grew in the highest linezolid or fosfomycin concentration were passaged five times on drug-free agars, and then their MICs were determined by the agar dilution methods to check for mutants and stored for further testing (Díez-Aguilar et al., 2015). All MPC studies were performed in three times.

\section{Characterization of Resistance Mechanisms}

Five original strains and their corresponding mutant derivatives recovered from the single-drug MPC studies were sequenced and compared. DNA was harvested using TIANamp bacteria DNA Kit (Tiangen, Beijing, China). The possible mechanisms of linezolid resistance were screened by polymerase chain reaction (PCR) using previously reported primers and conditions: the $23 \mathrm{~S}$ rRNA domain (Gawryszewska et al., 2017), ribosomal protein (L3 and L4) domain (Lee et al., 2017), the methyltransferase gene cfr (Doern et al., 2016), and ABC-type transporter gene optrA (Wang et al., 2015). Meanwhile, resistance genes related to fosfomycin ( $f o s B, M u r A, g l p T$, and $u h p T$ ) were also amplified by PCR (Fu et al., 2016; Zhang et al., 2019). All PCR positive products were subjected to sequencing analysis. The primers (listed in Supplementary Table 1) used for the sequencing reaction were the same as those used for PCR. The nucleotide sequences were compared with the E. faecalis ATCC29212 strain 
(no. CP008816.1) and E. faecium ZY11 strain (no. CP038995.1). The nucleotide sequence comparison was completed by BLAST ${ }^{1}$ and SnapGene Viewer (Version 5.1).

\section{Nucleotide Sequence Accession Numbers}

The sequences for strains the have been deposited in GenBank with the following accession numbers: GenBank accession MW301818-MW301829 (rplC and rplD gene related sequences); GenBank MW281777-MW281785 (23S RNA gene related sequences); GenBank MW357580-MW357581 (MurA gene related sequences).

\section{Statistical Analysis}

All statistical analyses were performed with GraphPad Prism, version 7.0 (GraphPad Software Inc., San Diego, CA, United States). One-way ANOVA was performed to assess the changes in MPC of linezolid or fosfomycin, used alone and in combination. $P$-values $<0.05$ were considered statistically significant.

\section{RESULTS}

\section{Antimicrobial Susceptibility Testing}

Among the 43 Enterococcus isolates, 37 isolates (86.0\%) were susceptible to linezolid, 23 isolates (53.0\%) were susceptible to fosfomycin. The MIC90 for linezolid and fosfomycin were 2 and $128 \mathrm{mg} / \mathrm{L}$, respectively (Supplementary Table 2).

\section{In vitro Synergy Testing With the Checkerboard Method}

The FICI values of all tested strains (Supplementary Table 2) illustrated that linezolid showed synergy or additivity in combination with fosfomycin against most of the tested strains (69.8\%). No antagonistic effect was detected against all isolates evaluated.

\section{MIC $_{99 \%}$ Alone, MPC of Single Drugs and Combinations}

For the five selected isolates with different FICI values, their $\mathrm{MIC}_{99 \%}$ alone, MPC alone of the two antimicrobial agents were listed on Table 1. For the five tested isolates, the MPCs of linezolid used alone ranged from 8.0 to $25.6 \mathrm{mg} / \mathrm{L}$, while MPC/MIC ratio was in the range of 3-6. The MPC value of fosfomycin used alone was 1228.8 to $2321.1 \mathrm{mg} / \mathrm{L}$, and the ratio of MPC/MIC was 13 to 20. However, linezolid combined with Fosfomycin can limit the enrichment of enterococcal resistant mutants at a lower concentration (Table 2). Furthermore, different proportions of linezolid and fosfomycin in a combination would present different MPCs. Compared with the MPC of linezolid or fosfomycin alone, the MPC of both two agents in combination were significantly reduced $(P<0.05)$ (Supplementary Figures 1, 2).

${ }^{1}$ http://blast.ncbi.nlm.nih.gov/Blast.cgi
TABLE 1 | MIC ${ }_{99 \%}$ s and MPCs of two antimicrobial agents alone for five selected Enterococcus strains.

\begin{tabular}{|c|c|c|c|c|c|c|c|}
\hline \multirow[t]{2}{*}{ Isolates } & \multicolumn{2}{|c|}{ MIC (mg/L) } & \multicolumn{2}{|c|}{$\mathrm{MIC}_{99 \%}(\mathrm{mg} / \mathrm{L})$} & \multicolumn{2}{|c|}{ MPC (mg/L) } & \multirow[t]{2}{*}{$\mathbf{F I C l}$} \\
\hline & LIN & FOS & LZD & FOS & LZD & FOS & \\
\hline NO.1 & 2 & 128 & 2.0 & 126.2 & 8.5 & 2321.1 & 0.625 \\
\hline NO.5 & 2 & 128 & 1.9 & 126.8 & 8.0 & 2321.1 & 0.5 \\
\hline NO.6 & 2 & 128 & 1.9 & 120.3 & 10.4 & 1774.9 & 0.375 \\
\hline NO.22 & 8 & 128 & 7.8 & 112.3 & 25.6 & 2321.1 & 0.312 \\
\hline $\mathrm{NO} .43$ & 2 & 64 & 1.9 & 64.0 & 8.5 & 1228.8 & 1.0 \\
\hline
\end{tabular}

MIC, minimum inhibitory concentration; MIC99\%, a minimal concentration that inhibits colony formation by 99\%; MPC, mutant prevention concentration; LZD, linezolid; FOS, fosfomycin; FICl, fractional inhibitory concentration index.

\section{SI Values of Two Drugs When Used Alone or Combined in Different Proportions}

The width of the mutant selection window (MSW) is termed as selection index (SI), which can be expressed as the ratio of MPC to MIC $99 \%$. Closing MSW implied that SI were less than or equal to one. Based on the MSW theory, a combination in which SI of each agent was less than or equal to one would be efficacious to prevent antimicrobial resistance. The SI values of the two agents used alone or in combination with different proportions were showed in Figure 1. It can be clearly observed from the Figure 1: (1) For each strain, the SI values of fosfomycin alone were significantly higher than that of linezolid alone. It means that the MSW of Fosfomycin was larger than the MSW of linezolid. However, the MSW of fosfomycin was firstly to be closed when combined with linezolid. (2) Linezolid combined with fosfomycin in different ratios, the SI values of the two were different. (3) For the isolates with the value of FICI $\leq 0.5$ (NO.22, NO.6, and NO.5), the combination of linezolid and fosfomycin can simultaneously close each other's MSW within a certain ratio rang. The smaller FICI, the wider range of the combination to close each other's mutation selection window (Figures 1B-D). But, for the NO.1 and NO.43 strain (with FICI > 0.5), in any composition ratio, the combination of linezolid and fosfomycin cannot shut down each other's MSW at the same time, (Figures 1A,E). Above experimental data and analyses showed that a roughly positive correlations between SI and FICI suggested that the smaller the FICI value of linezolid and fosfomycin was, the more probable the combination was to close each other's MSW (SI was less than or equal to one).

\section{Characterization of Linezolid or Fosfomycin Resistance Mechanisms}

The sequencing results and MIC determination results of the resistant mutants and its parent strains were presented in Tables 3, 4. As shown in Table 3, linezolid-resistant mutants showed low-level resistance to linezolid, and its MIC value was 8 to $32 \mathrm{mg} / \mathrm{L}$. Mutations in the $\mathrm{rplC}$ gene encoding ribosomal protein L3 or the rplD gene encoding ribosomal protein L4 were detected in the linezolid-resistant mutants of each strain (Table 3). Nucleotide substitutions of nt608, nt609, nt613, nt614 led to substitution of Glu by Gly at amino acid of rplD. In 
TABLE 2 | MPCs of linezolid and fosfomycin in combinations with thirteen different proportions.

\begin{tabular}{|c|c|c|c|c|c|c|c|c|c|c|c|c|c|}
\hline \multirow[t]{2}{*}{ Isolates } & \multicolumn{13}{|c|}{ MPCs of two antimicrobial agents in combinations with thirteen different proportions (mg/L) (LZD: FOS) } \\
\hline & $64: 1$ & $32: 1$ & $16: 1$ & $8: 1$ & $4: 1$ & $2: 1$ & $1: 1$ & $1: 2$ & $1: 4$ & $1: 8$ & $1: 16$ & $1: 32$ & $1: 64$ \\
\hline NO.1 & $6.9 / 0.11$ & $6.4 / 0.2$ & $5.9 / 0.4$ & $5.3 / 0.7$ & $3.7 / 0.9$ & $3.5 / 1.5$ & $3.5 / 3.5$ & $2.7 / 5.9$ & $5.3 / 21.3$ & $4.8 / 38.4$ & $3.5 / 55.5$ & $2.7 / 172.5$ & $2.0 / 128.0$ \\
\hline NO.5 & 6.9/0.11 & $5.3 / 0.17$ & $5.3 / 0.3$ & $6.4 / 0.8$ & $5.3 / 1.3$ & $5.3 / 2.7$ & $3.2 / 3.2$ & $2.9 / 5.9$ & $4.8 / 19.2$ & $2.9 / 23.5$ & $2.7 / 42.7$ & 2.9/93.9 & $1.9 / 119.5$ \\
\hline NO.6 & 6.9/0.11 & $5.9 / 0.18$ & 3.2/0.2 & $2.7 / 0.3$ & 2.4/0.6 & 2.7/1.3 & $2.4 / 2.4$ & $2.4 / 4.8$ & 4.8/19.2 & $2.7 / 21.3$ & $1.6 / 27.7$ & $1.6 / 51.2$ & $1.7 / 119.5$ \\
\hline NO.22 & 21.3/0.33 & 21.3/0.67 & 21.3/1.3 & $20.3 / 2.5$ & $18.1 / 4.5$ & $17.1 / 8.5$ & $14.9 / 14.9$ & $6.4 / 12.8$ & $6.9 / 27.7$ & $6.4 / 51.2$ & $6.9 / 110.9$ & $5.9 / 187.7$ & $5.9 / 375.5$ \\
\hline NO.43 & $6.4 / 0.1$ & $5.9 / 0.18$ & $5.9 / 0.37$ & 4.8/0.6 & 5.3/1.3 & $4.8 / 2.4$ & $4.8 / 4.8$ & $3.7 / 7.5$ & $3.2 / 11.7$ & $2.4 / 19.2$ & $2.4 / 38.4$ & 2.9/93.9 & $1.9 / 119.5$ \\
\hline
\end{tabular}

MPC, mutant prevention concentration; LZD, linezolid; FOS, fosfomycin.

the NO.1-LM strain, a mutation of nt610 $(\mathrm{A} \rightarrow \mathrm{T})$ and nt610 $(\mathrm{T} \rightarrow \mathrm{C}$ ) resulted the amino acid changes of rplC. Moreover, four of five linezolid resistant mutants presented mutations both in $r p l C$ and $r p l D$ gene. Except for the detection of the $r p l C$ gene mutation encoding ribosomal protein L3 in the NO.22 strain, no gene mutation was detected in the other parental strains. As fosfomycin mutant derivatives (Table 4), compared with their parent strains, four of five showed highly resistant to fosfomycin (MICs rang 1024 to $2048 \mathrm{mg} / \mathrm{L}$ ). The sequencing results showed that a nucleotide substitution at nt465 $(\mathrm{A} \rightarrow \mathrm{G})$ in strain NO.1-FM and at $\mathrm{nt} 1163(\mathrm{~A} \rightarrow \mathrm{C}), \mathrm{nt1216}(\mathrm{T} \rightarrow \mathrm{A})$ in strain NO.5-FM, resulted the amino acid changes of MurA. However, some fosfomycin mutants and their parents did not successfully amplify several drug-resistant gene related fragments.

\section{DISCUSSION}

In this study, the FICI values of 43 strains indicated that linezolid-fosfomycin combination showed synergistic or additive effect on $69.8 \%$ of the tested strains and no antagonistic effects was observed. Consistent with this experiment, the synergistic antibacterial activity of the linezolid-fosfomycin combination against Staphylococcus aureus and Enterococcus was also confirmed in vitro time-killing curve (Chai et al., 2016; Chen et al., 2018; Qi et al., 2019). However, research on the combination to prevent resistance was still lacking.

Mutant prevention concentration and mutant selection window are special parameters that may provide useful information about the necessary drug concentration required in the infection area, in order to avoid the emergence of resistance, particular in case of high bacterial load (Vassilara et al., 2017). For the five strains, the MPCs of fosfomycin alone were 13 to 20-fold than their MICs, which implied that the MSW of fosfomycin was very wider. This results also showed in the MPC study of fosfomycin against Staphylococcus aureus (Mei et al., 2015), Escherichia coli (Pan et al., 2017), and Pseudomonas aeruginosa (Díez-Aguilar et al., 2015). Moreover, all MPC values of five selected strains exceed $1000 \mathrm{mg} / \mathrm{L}$. However, according to the pharmacokinetic study of fosfomycin, a 4-g intravenous infusion reaches $\mathrm{C}_{\max }$ (peak concentration) of $200-250 \mathrm{mg} / \mathrm{L}$ and an 8-g dose $C_{\max }$ of 260-450 mg/L (Roussos et al., 2009). It means that when fosfomycin monotherapy was used, the concentration at the infected site easily falls into the MSW, which may cause to the occurrence of bacterial resistance. The paradox is that fosfomycin resistance develops readily in vitro but less so in vivo (Falagas et al., 2016). The apparent discrepancy between in vitro and in vivo may partly explained by the function of immune system. Handel et al. revealed that an immune response greatly narrows the MSW and decreases the emergence of resistance despite a large drug-induced decline of bacteria numbers (Handel et al., 2009). Concern about the clinical application of fosfomycin is that resistance may appear during monotherapy. Currently, Fosfomycin is generally recommended in combination with other antibacterial drugs to treat bacterial infections (Falagas et al., 2018). As the results of linezolid MPC alone, the MPC values were 8.0 to $10.4 \mathrm{mg} / \mathrm{L}$ in the four linezolid-susceptible isolates (NO.1, NO.5, NO.6, and NO.43). This is a slightly higher than the MPC of clinically isolated enterococci reported in other studies (Zinner et al., 2008; Allen and Bierman, 2009). Some studies showed that maintaining drug concentrations above its MPC throughout therapy can severely restrict the acquisition of linezolid resistant mutants (Zinner et al., 2018; Alieva et al., 2019). The in vitro pharmacodynamics of linezolid against a clinical isolate of E. faecium (MIC1.8 mg/L and MPC $7 \mathrm{mg} / \mathrm{L})$ demonstrated that an AUC24/MIC ratio $>200 \mathrm{~h}\left(\mathrm{AUC}_{24}, 24 \mathrm{~h}\right.$ area under the curve) was estimated to restrict the selection of linezolid-resistant enterococci (Zinner et al., 2008). However, this estimated value is twice the value provided by a $600 \mathrm{mg}$ clinical dose of twice-daily linezolid (Zinner et al., 2008). Increasing the dose of linezolid can achieve its therapeutic effect, while this will increase the risk of adverse and toxic effects. Therefore, linezolid monotherapy may be not a wise choice to prevent bacterial resistance and improve drug safety.

Combinations including individual drug constituents with smaller MSWs may have better ability in preventing the evolution of resistance ( $\mathrm{Ni}$ et al., 2016). For the five tested strains, the MSW of fosfomycin monotherapy was much larger than that of linezolid. Interestingly, the MSW of fosfomycin was prior to be closed in the two agents of the combination. Furthermore, the combination of linezolid and fosfomycin in different ratios can effectively suppress the enrichment of enterococcal resistant mutants at a lower concentration. Compared with linezolid or fosfomycin alone, the MPC values of the two antibacterial drugs were significantly reduced when the two drugs combined in different proportions $(P<0.05)$. It may suggest that the MSW of one antimicrobial agent in combination can be narrowed or even close by increasing the proportion of another agent whether it's synergy or not. This has also confirmed by many previous studies 
A

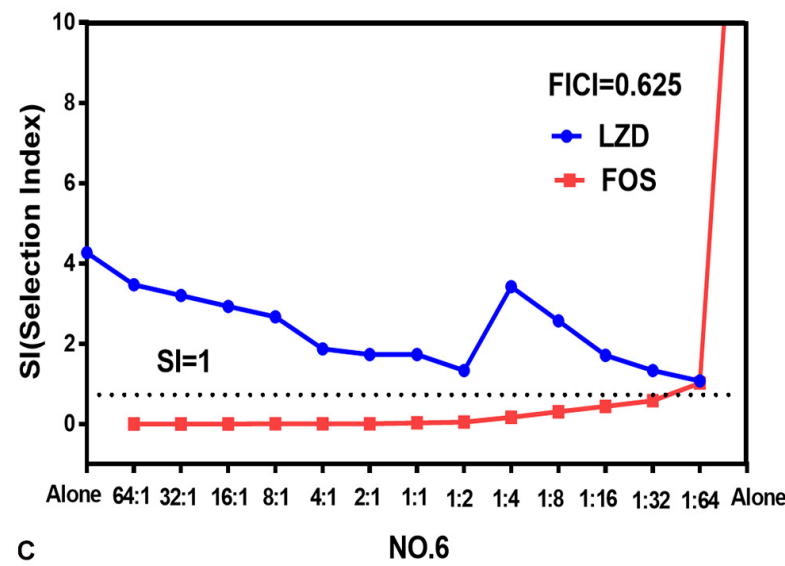

C

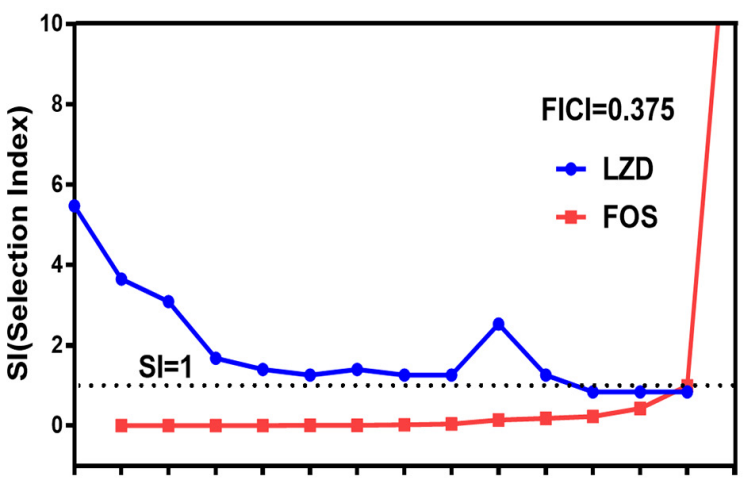

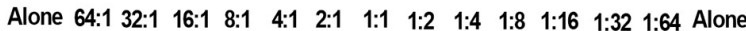

B
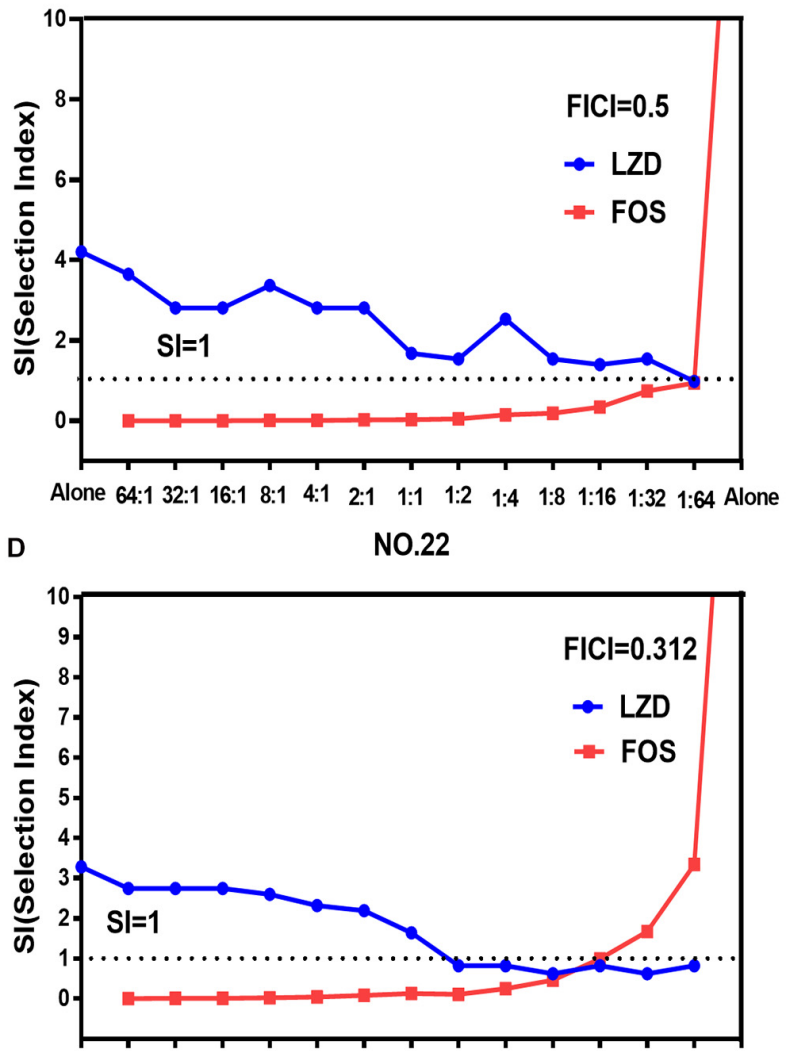

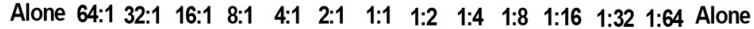

E

N0.43

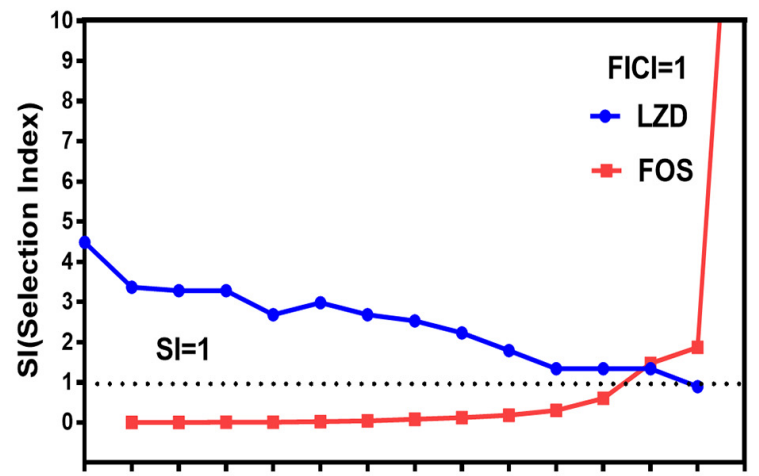

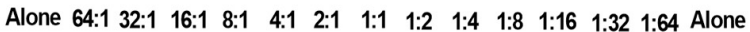

FIGURE 1 | Mutant selection indexes (SIs) of linezolid and fosfomycin when the two agents used alone or in combination with different ratios (linezolid: fosfomycin) against five enterococci. (A) NO.1 strain; (B) NO.5 strain; (C) NO.6 strain; (D) NO.22 strain; (E) NO.43 strain; LZD, linezolid; FOS, fosfomycin; FICl, fractional inhibitory concentration index; that the Sls of two agents in a combination including thirteen proportions were simultaneously less than or equal to one represented their MSWs were closed each other.

on combinations such as minocycline and amikacin (Wentao et al., 2018) or gentamicin, tigecycline and amikacin (Ni et al., 2016), fosfomycin and tobramycin (Díez-Aguilar et al., 2015). However, something may be different when it is discussed that the combination of linezolid and fosfomycin simultaneously closes each other's mutation selection window. Closing MSW mean that mutant selection index (SI, the ratio of MPC to MIC99\%) were less than or equal to one. For the selected isolates with FICI $\leq 0.5$ (NO.5, NO.6, and NO.22), both the SI of linezolid and fosfomycin in combination were simultaneously less than or equal to one within a certain range of proportions (Figures 1B-D). In addition, the more significant synergistic effect between linezolid 
TABLE 3 | MIC values of linezolid and resistance mechanisms in linezolid resistant-mutants obtained from the MPC study of linezolid alone.

\begin{tabular}{|c|c|c|c|c|c|c|}
\hline \multirow[t]{2}{*}{ Isolates } & \multicolumn{5}{|c|}{ Resistance gene } & \multirow[t]{2}{*}{ Linezolid MIC (mg/L) } \\
\hline & $r p / C$ & $r p I D$ & $c f r$ & optrA & 23s RNA & \\
\hline NO.1 & - & - & - & - & - & 2 \\
\hline NO.1-LM & A610T(Lys192Asn)/T611C(Ser193Pro) /615A(194Ser) & Insert(610A)/A613G(Glu166Gly) & - & - & - & 16 \\
\hline NO.5 & - & - & - & - & - & 2 \\
\hline NO.5-LM & T18A(lle6Asn)/T604C/T607A & Insert(610A/T611)/A614G(Glu167Gly) & - & - & - & 8 \\
\hline NO.6 & - & - & - & - & - & 2 \\
\hline NO.6-LM & - & A609G(Glu165Gly) & - & - & - & 8 \\
\hline NO.22 & A606T/insert(8C/9A) & - & - & - & - & 8 \\
\hline NO.22-LM & A605T/insert(8C) & A608G(Glu165Gly) & - & - & - & 32 \\
\hline $\mathrm{NO} .43$ & - & - & - & - & - & 2 \\
\hline NO.43-LM & A605T & С349T & - & - & - & 8 \\
\hline
\end{tabular}

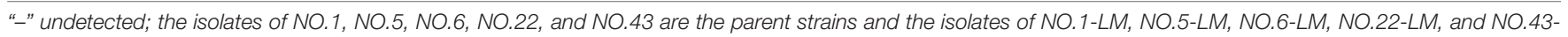

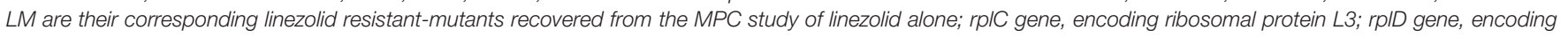
ribosomal protein L4.

and fosfomycin was, the wider extent of the two drugs closing their MSW was. But for the tested strains with FICI $>0.5$ (NO.1 and NO.43), the two agents in combination cannot close each other's mutation selection window at the same time in any composition ratio. Based on the above results (Figures 1A-E), we found a rough positive correlation between SI and FICI, which displayed that the smaller FICI was, the more probable the combination was to close each other's MSW (SI was less than or equal to one). Similar to our results, Xu et al. (2018) revealed that the smaller FICIs of two agents in combinations were, the more probable their MSWs were to close each other. In accordance with the MSW theory, the synergistic combination of linezolid and fosfomycin simultaneously closing their MSW has a great potency to prevent enterococcal resistance.

Could the synergistic combination of linezolid and fosfomycin completely prevent resistance? Combination efficacy was

TABLE 4 | MIC values of fosfomycin and resistance mechanisms in fosfomycin resistant-mutants obtained from the fosfomycin MPC study.

\begin{tabular}{|c|c|c|c|c|c|}
\hline \multirow[t]{2}{*}{ Isolates } & \multicolumn{4}{|c|}{ Resistance gene } & \multirow{2}{*}{$\begin{array}{l}\text { Fosfomycin } \\
\text { MIC (mg/L) }\end{array}$} \\
\hline & MurA & uhpT & $g / p T$ & fos $B$ & \\
\hline NO.1 & - & - & - & - & 128 \\
\hline NO.1-FM & A465G(Cys210Arg)/C754T/ & - & - & - & 2048 \\
\hline NO.5 & - & - & - & - & 128 \\
\hline NO.5-FM & $\begin{array}{l}\text { A1163C(Leu344Val)/ } \\
\text { T1216A(Asp361Val)/C1091T }\end{array}$ & - & - & - & 2048 \\
\hline NO.6 & - & - & - & - & 128 \\
\hline NO.6-FM & - & - & - & - & 1024 \\
\hline NO.22 & - & - & - & - & 128 \\
\hline NO.22-FM & - & - & - & - & 512 \\
\hline NO.43 & - & - & - & - & 128 \\
\hline NO.43-FM & - & - & - & - & 1024 \\
\hline
\end{tabular}

"-" undetected; the isolates of NO.1, NO.5, NO.6, NO.22, and NO.43 are the parent strains and the isolates of NO.1-FM, NO.5-FM, NO.6-FM, NO.22-FM, and NO.43-FM are their corresponding fosfomycin resistant-mutants obtained from the MPC study of fosfomycin alone. complicated by many factors, including the proportion of drugs in the combination. Although a lot of combinations have been reported to prevent bacterial resistance, while different proportions of two drugs in combination rarely determined. In this study, thirteen proportions of linezolid and fosfomycin in combination was designed and the results show that different ratios of linezolid and fosfomycin in a combination would present different MPCs and SIs. Taking into account the difference in the pharmacokinetics of linezolid and fosfomycin in vivo, the ratios of two drugs in blood and infectious sites may be different even if the two agents administrated at fixed ratio, which would result in different effects in preventing resistance (Xu et al., 2018). Therefore, it is preferable to select a combination of two antimicrobials that can close each other's mutation selection window in a wide range of proportions. However, even though the linezolid-fosfomycin combination presented evidently synergistic activities against enterococcus NO.22 and NO.6, only proportions against NO.22 (1:2 to 1:16) and NO.6 (1:16 to 1:64) could close each other's MSW (Figures 1C,D). Thereby, it was best to choose the combination with the small FICI value as much as possible to prevent enterococcal resistance. Ideally, the maximum FICI value was better less than 0.5 .

The common mechanisms of Enterococcus resistance to linezolid include point mutations in chromosome 23S rRNA genes or genes encoding L3, L4, and L22 ribosomal proteins (Mendes et al., 2014; Wang et al., 2014). Other important mechanisms include plasmid-mediated chloramphenicolflorfenicol resistance cfr gene or ribosome protection gene optrA and poxtA (Wang et al., 2014; Park et al., 2020; Ruiz-Ripa et al., 2020). Correspondingly, several mechanisms have been proposed to be related to fosfomycin resistance including fosfomycin forming an inactive adduct (Falagas et al., 2018), fosfomycin modification enzyme (Cassir et al., 2014), mutations in the chromosomal genes encoding fosfomycin transporters ( $\mathrm{Fu}$ et al., 2016; Xu et al., 2017) and mutations in the target enzyme MurA (Falagas et al., 2016). In order to explore the resistance mechanism of linezolid resistant mutants or fosfomycin resistant 
mutants, several related genes were amplified in this experiment. All linezolid resistant mutants showed low levels resistance to linezolid. Moreover, only mutations in $r p l C$ gene encoding ribosomal proteins $\mathrm{L} 3$ or $r p l D$ gene encoding ribosomal proteins L4 were detected in the linezolid resistant mutants. The outcomes are in accord with recent study which reported that the L3 and L4 mutations are associated with low-level linezolid resistance in enterococci (Chen et al., 2013). Differently, Hua et al. (2019) held that since the L3 and L4 mutations did not simultaneously occur in the same strain, they play a negligible role in linezolid resistance. But, in this study, four linezolid resistant mutants had both ribosomal protein L3 and L4 mutations. For the mechanism of resistance to fosfomycin, sequencing analyses detected distinct mutations in the MurA gene of the two fosfomycin resistant mutants. The bacterial enzyme MurA catalyzes the transfer of enolpyruvate from Phosphoenolpyruvate (PEP) to uridine diphospho-N-acetylglucosamine (UNAG), which is the first step of bacterial cell wall biosynthesis (Kurnia et al., 2019). The mutations detected in MurA have been shown to reduce the affinity of fosfomycin (Fu et al., 2016; Xu et al., 2017). Recently study shown that the mutations in the fosfomycin target enzyme MurA were related to the resistance mechanisms clinically isolated enterococci (Zhang et al., 2019). However, the mechanism of three fosfomycin resistant mutants to fosfomycin remains unclear. The mechanism that governs fosfomycin resistance in enterococci requires further study.

\section{Limitations of This Study}

Firstly, although different ratios of linezolid and fosfomycin were designed in this study, the static concentration in vitro could not truly reflect the dynamic process of the drug in vivo. And we neglected the influence of in vivo immunity on resistance selection. The MSW hypothesis and the MPC concept have been applied to a planktonic mode of bacterial growth and not for biofilms, which are one of the causes of bacterial resistance (Cantón and Morosini, 2011). These findings need to be further verified in dynamic model of pharmacokinetics and pharmacodynamics in vitro (Golikova et al., 2017), animal models (Pan et al., 2017), bacterial biofilm (Siala et al., 2018; Sharma et al., 2019c). Secondly, only five strains of Enterococcus were used in MSW and MPC studies. Thirdly, mutations in drug-resistant gene from chromosome preliminarily verified the applicability of MSW theory to linezolid and fosfomycin. However, under clinical conditions, enterococci can acquire resistance via chromosomal mutations and lateral gene transfer. Our study could not reflect the entire complex clinical situation, because MPCs/MSWs derived from chromosomal mutations but not lateral gene transfer ( $\mathrm{Ni}$ et al., 2016). Furthermore, the relationship between a mutation and drug resistance is not always a simple one-to-one correspondence. Although many mutations contributing to antibiotic resistance have been identified, the relationship between the mutations and the related phenotypic changes in charge of resistance has yet to be fully elucidated (Suzuki et al., 2014). Taking into,account the complexity of bacterial resistance mechanisms, it is necessary to use proteomics (Vranakis et al., 2014; Yan et al., 2018) and other methods (Hua et al., 2018) to further elaborate the specific mechanisms.

\section{CONCLUSION}

Linezolid combined with fosfomycin could validly restrict the enrichment of resistant enterococci at low concentrations, compared with the two drugs alone. The synergistic combination of linezolid and fosfomycin may have better ability in preventing the evolution of resistance in clinic and provide a new option for clinical treatment of enterococcal infection.

\section{DATA AVAILABILITY STATEMENT}

The datasets presented in this study can be found in online repositories. The names of the repository/repositories and accession number(s) can be found below: GenBank, accession: MW301818-MW301829, MW281777-MW281785, and MW357580-MW357581.

\section{AUTHOR CONTRIBUTIONS}

$\mathrm{XH}$ and JL conceived the idea and designed the study. LJ performed the study, analyzed the data, and wrote the manuscript. NX, MC, and YL provided technical support. SW and JM revised the manuscript. All authors read and approved the final version of the manuscript.

\section{FUNDING}

This study was supported by the National Natural Science Foundation of China (81173133), the Fund of Excellent Talents in Colleges and Universities of Anhui Province, China (gxbjZD06), and the Fund of Academic Leaders of Anhui Province, China (2015D068).

\section{ACKNOWLEDGMENTS}

We thank Department of Infectious Diseases, The First Affiliated Hospital of Anhui Medical University for its assistance.

\section{SUPPLEMENTARY MATERIAL}

The Supplementary Material for this article can be found online at: https://www.frontiersin.org/articles/10.3389/fmicb.2020. 605962/full\#supplementary-material

Supplementary Figure 1 | The MPC of linezolid when it used alone or combined with fosfomycin in thirteen proportions (linezolid: fosfomycin) against five enterococci. (A) NO.1 strain; (B) NO.5 strain; (C) NO.6 strain; (D) NO.22 strain; (E) NO.43 strain; MPC, mutant prevention concentration; ${ }^{*} p$-value $<0.05$; ${ }^{\star *} p$-value $<0.001 ;{ }^{* \star \star} p$-value $<0.0001$.

Supplementary Figure 2 | The MPC of fosfomycin when it used alone or combined with linezolid in thirteen proportions (linezolid: fosfomycin) against five enterococci. (A) NO.1 strain; (B) NO.5 strain; (C) NO.6 strain; (D) NO.22 strain; (E) NO.43 strain; MPC, mutant prevention concentration; ${ }^{* \star *} p$-value $<0.0001$. 


\section{REFERENCES}

Alieva, K. N., Golikova, M. V., Portnoy, Y. A., Dovzhenko, S. A., Kobrin, M. B., Zinner, S. H., et al. (2019). Concentration-dependent enrichment of resistant Enterococcus faecium exposed to linezolid in an in vitro dynamic model. J. Chemother. 30, 364-370. doi: 10.1080/1120009x.2018.1533267

Alieva, K. N., Strukova, E. N., Golikova, M. V., Portnoy, Y. A., Zinner, S. H., and Firsov, A. A. (2018). Time inside the mutant selection window as a predictor of staphylococcal resistance to linezolid. J. Antibiot. 71, 514-521. doi: 10.1038/ s41429-017-0016-9

Allen, G. P., and Bierman, B. C. (2009). In vitro analysis of resistance selection by linezolid in vancomycin-susceptible and -resistant Enterococcus faecalis and Enterococcus faecium. Int. J. Antimicrob. Agents 34, 21-24. doi: 10.1016/ j.ijantimicag.2008.12.011

Bai, B., Hu, K., Zeng, J., Yao, W., Li, D., Pu, Z., et al. (2019). Linezolid consumption facilitates the development of linezolid resistance in Enterococcus faecalis in a tertiary-care hospital: a 5-year surveillance study. Microb. Drug Resist. 25, 791-798. doi: 10.1089/mdr.2018.0005

Bayram, N., Duzgol, M., Kara, A., Ozdemir, F. M., and Devrim, I. (2017). Linezolidrelated adverse effects in clinical practice in children. Arch. Argent. Pediatr. 115, 470-475. doi: 10.5546/aap.2017.eng.470

Blondeau, J. M. (2009). New concepts in antimicrobial susceptibility testing: the mutant prevention concentration and mutant selection window approach. Vet. Dermatol. 20, 383-396. doi: 10.1111/j.1365-3164.2009.00856 x

Cantón, R., and Morosini, M.-I. (2011). Emergence and spread of antibiotic resistance following exposure to antibiotics. FEMS Microbiol. Rev. 35, 977-991. doi: 10.1111/j.1574-6976.2011.00295.x

Cassir, N., Rolain, J., and Brouqui, P. (2014). A new strategy to fight antimicrobial resistance: the revival of old antibiotics. Front. Microbiol. 5:551. doi: 10.3389/ fmicb.2014.00551

Chai, D., Liu, X., Wang, R., Bai, Y., and Cai, Y. (2016). Efficacy of linezolid and fosfomycin in catheter-related biofilm infection caused by methicillin-resistant Staphylococcus aureus. Biomed. Res. Int. 2016, 1-7. doi: 10.1155/2016/641 3982

Chen, H., Li, L., Liu, Y., Wu, M., Xu, S., Zhang, G., et al. (2018). In vitro activity and post-antibiotic effects of linezolid in combination with fosfomycin against clinical isolates of Staphylococcus aureus. Volume 11, 2107-2115. doi: 10.2147/ IDR.S175978

Chen, H., Wu, W., Ni, M., Liu, Y., Zhang, J., Xia, F., et al. (2013). Linezolid-resistant clinical isolates of enterococci and Staphylococcus cohnii from a multicentre study in China: molecular epidemiology and resistance mechanisms. Int. J. Antimicrob. Agents 42, 317-321. doi: 10.1016/j.ijantimicag.2013.06.008

Clsi. (2019). Performance Standards for Antimicrobial Susceptibility Testing; Twenty-ninth Informational Supplement (M100). Wayne, PA: Clinical and Laboratory Standards Institute.

Davis, H., Brown, R., Ashcraft, D., and Pankey, G. (2020). In vitro synergy with fosfomycin plus doxycyclin against linezolid and vancomycin-resistant Enterococcus faecium. J. Global Antimicrob. Resist. 22, 78-83. doi: 10.1016/j.jgar. 2020.01.014

Díez-Aguilar, M., Morosini, M. I., Tedim, A. P., Rodríguez, I., Aktaş, Z., Cantón, R. et al. (2015). Antimicrobial activity of fosfomycin-tobramycin combination against Pseudomonas aeruginosa isolates assessed by time-kill assays and mutant prevention concentrations. Antimicrob. Agents Chemother. 59, 60396045. doi: 10.1128/AA

Doern, C. D., Park, J. Y., Gallegos, M., Alspaugh, D., and Burnham, C. D. (2016). Investigation of linezolid resistance in Staphylococci and Enterococci. J. Clin. Microbiol. 54, 1289-1294. doi: 10.1128/JCM.01929-15

Drago, L., Nicola, L., and De Vecchi, E. (2008). A comparative in-vitro evaluation of resistance selection after exposure to teicoplanin, vancomycin, linezolid and quinupristin-dalfopristin in Staphylococcus aureusan Enterococcus spp. Clin. Microbiol. Infect. 14, 608-611. doi: 10.1111/j.1469-0691.2008.01993.x

Drlica, K. (2003). The mutant selection window and antimicrobial resistance. J. Antimicrob. Chemother. 52, 11-17. doi: 10.1093/jac/dkg269

Drlica, K., and Zhao, X. (2007). Mutant selection window hypothesis updated. Clin. Infect. Dis. 44, 681-688. doi: 10.1086/511642

Durão, P., Balbontín, R., and Gordo, I. (2018). Evolutionary mechanisms shaping the maintenance of antibiotic resistance. Trends Microbiol. 26, 677-691. doi: 10.1016/j.tim.2018.01.005
Falagas, M. E., Athanasaki, F., Voulgaris, G. L., Triarides, N. A., and Vardakas, K. Z. (2018). Resistance to fosfomycin: Mechanisms, Frequency and Clinical Consequences. Int. J. Antimicrob. Agents 53, 22-28. doi: 10.1016/j.ijantimicag. 2018.09.013

Falagas, M. E., Lourida, P., Poulikakos, P., Rafailidis, P. I., and Tansarli, G. S. (2014). Antibiotic treatment of infections due to carbapenem-resistant Enterobacteriaceae: systematic evaluation of the available evidence. Antimicrob. Agents Chemother. 58, 654-663. doi: 10.1128/AAC.01222-13

Falagas, M. E., Vouloumanou, E. K., Samonis, G., and Vardakas, K. Z. (2016). Fosfomycin. Clin. Microbiol. Rev. 29, 321-347. doi: 10.1128/CMR.00068-15

Fiore, E., Van Tyne, D., and Gilmore, M. S. (2019). Pathogenicity of Enterococci. Microbiol. Spectr. 7, 31298205. doi: 10.1128/microbiolspec.GPP3-0053-2018

Fu, Z., Ma, Y., Chen, C., Guo, Y., Hu, F., Liu, Y., et al. (2016). Prevalence of fosfomycin resistance and mutations in murA, glpT, and $u h p T$ in methicillinresistant Staphylococcus aureus strains isolated from blood and cerebrospinal fluid samples. Front. Microbiol. 6:1544. doi: 10.3389/fmicb.2015.01544

García-Solache, M., and Rice, L. B. (2019). The Enterococcus: a model of adaptability to its environment. Clin. Microbiol. Rev. 32, e18-e58. doi: 10.1128/ CMR.00058-18

Gawryszewska, I., żabicka, D., Hryniewicz, W., and Sadowy, E. (2017). Linezolidresistant enterococci in Polish hospitals: species, clonality and determinants of linezolid resistance. Eur. J. Clin. Microbiol. 36, 1279-1286. doi: 10.1007/s10096017-2934-7

Gilmore, M. S., Lebreton, F., and van Schaik, W. (2013). Genomic transition of enterococci from gut commensals to leading causes of multidrug-resistant hospital infection in the antibiotic era. Curr. Opin. Microbiol. 16, 10-16. doi: 10.1016/j.mib.2013.01.006

Golikova, M. V., Strukova, E. N., Portnoy, Y. A., Dovzhenko, S. A., Kobrin, M. B., Zinner, S. H., et al. (2017). Predicting effects of antibiotic combinations using MICs determined at pharmacokinetically derived concentration ratios: in vitro model studies with linezolid- and rifampicin-exposed Staphylococcus aureus. J. Chemother. 29, 267-273. doi: 10.1080/1120009X.2017.1281093

Haghi, F., Lohrasbi, V., and Zeighami, H. (2019). High incidence of virulence determinants, aminoglycoside and vancomycin resistance in enterococci isolated from hospitalized patients in northwest iran. BMC Infect. Dis. 19:744. doi: 10.1186/s12879-019-4395-3

Handel, A., Margolis, E., and Levin, B. R. (2009). Exploring the role of the immune response in preventing antibiotic resistance. J. Theor. Biol. 256, 655-662. doi: 10.1016/j.jtbi.2008.10.025

Hua, R., Xia, Y., Wu, W., Yan, J., and Yang, M. (2018). Whole transcriptome analysis reveals potential novel mechanisms of low-level linezolid resistance in Enterococcus faecalis. Gene 647, 143-149. doi: 10.1016/j.gene.2018.01.008

Hua, R., Xia, Y., Wu, W., Yang, M., and Yan, J. (2019). Molecular epidemiology and mechanisms of 43 low-level linezolid-resistant Enterococcus faecalis strains in chongqing. China. Ann. Lab. Med. 39, 36-42. doi: 10.3343/alm.2019.39.1.36

Kurnia, D., Apriyanti, E., Soraya, C., and Satari, M. H. (2019). Antibacterial flavonoids against oral bacteria of Enterococcus Faecalis ATCC 29212 from Sarang semut (myrmecodia pendans) and its inhibitor activity against enzyme mura. Curr. Drug Discov. Technol. 16, 290-296. doi: 10.2174/ 1570163815666180828113920

Lee, E. Y., and Caffrey, A. R. (2018). Thrombocytopenia with tedizolid and linezolid. Antimicrob. Agents Chemother. 62, e1417-e1453. doi: 10.1128/AAC. 01453-17

Lee, S., Huh, H. J., Song, D. J., Shim, H. J., Park, K. S., Kang, C., et al. (2017). Resistance mechanisms of linezolid-nonsusceptible enterococci in Korea: low rate of 23S rRNA mutations in Enterococcus faecium. J. Med. Microbiol. 66, 1730-1735. doi: 10.1099/jmm.0.000637

Leone, S., Noviello, S., and Esposito, S. (2016). Combination antibiotic therapy for the treatment of infective endocarditis due to enterococci. Infection 44, 273-281. doi: 10.1007/s15010-015-0836-0

Mei, Q., Ye, Y., Zhu, Y. L., Cheng, J., Chang, X., Liu, Y. Y., et al. (2015). Testing the mutant selection window hypothesis in vitro and in vivo with Staphylococcus aureus exposed to fosfomycin. Eur. J. Clin. Microbiol. 34, 737-744. doi: 10.1007/ s10096-014-2285-6

Mendes, R. E., Deshpande, L. M., and Jones, R. N. (2014). Linezolid update: stable in vitro activity following more than a decade of clinical use and summary of associated resistance mechanisms. Drug Resist. Updat. 17, 1-12. doi: 10.1016/j. drup.2014.04.002 
Mercuro, N. J., Davis, S. L., Zervos, M. J., and Herc, E. S. (2018). Combatting resistant enterococcal infections: a pharmacotherapy review. Expert Opin. Pharmaco. 19, 979-992. doi: 10.1080/14656566.2018.1479397

Ni, W., Cai, X., Wei, C., Di, X., Cui, J., Wang, R., et al. (2015). Efficacy of polymyxins in the treatment of carbapenem-resistant Enterobacteriaceae infections: a systematic review and meta-analysis. Braz. J. Infect. Dis. 19, 170-180. doi: 10.1016/j.bjid.2014.12.004

Ni, W., Wei, C., Zhou, C., Zhao, J., Liang, B., Cui, J., et al. (2016). Tigecyclineamikacin combination effectively suppresses the selection of resistance in clinical isolates of KPC ProducingKlebsiella pneumoniae. Front. Microbiol. 7:1304. doi: 10.3389/fmicb.2016.01304

Pan, A. J., Mei, Q., Ye, Y., Li, H. R., Liu, B., and Li, J. B. (2017). Validation of the mutant selection window hypothesis with fosfomycin against Escherichia coli and Pseudomonas aeruginosa: an in vitro and in vivo comparative study. J. Antibiot. 70, 166-173. doi: 10.1038/ja.2016.124

Park, K., Jeong, Y. S., Chang, J., Sung, H., and Kim, M. (2020). Emergence of optrA -mediated linezolid-nonsusceptible Enterococcus faecalis in a tertiary care hospital. Ann. Lab. Med. 40, 321-325. doi: 10.3343/alm.2020. 40.4.321

Qi, C., Xu, S., Wu, M., Zhu, S., Liu, Y., Huang, H., et al. (2019). Pharmacodynamics of linezolid-plus-fosfomycin against vancomycin-susceptible and -resistant Enterococci in vitro and in vivo of a galleria mellonella larval infection model. Volume 12, 3497-3505. doi: 10.2147/IDR.S219117

Roussos, N., Karageorgopoulos, D. E., Samonis, G., and Falagas, M. E. (2009). Clinical significance of the pharmacokinetic and pharmacodynamic characteristics of fosfomycin for the treatment of patients with systemic infections. Int. J. Antimicrob. Agents 34, 506-515. doi: 10.1016/j.ijantimicag. 2009.08.013

Ruiz-Ripa, L., Feßler, A. T., Hanke, D., Eichhorn, I., Azcona-Gutiérrez, J. M., Pérez-Moreno, M. O., et al. (2020). Mechanisms of linezolid resistance among enterococci of clinical origin in spain-detection of optrA- and cfr(D)-carrying e. faecalis. Micro Organ. 8:1155. doi: 10.3390/microorganisms 8081155

Sassi, M., Guérin, F., Zouari, A., Beyrouthy, R., Auzou, M., Fines-Guyon, M., et al. (2019). Emergence of optrA-mediated linezolid resistance in enterococci from France, 2006-16. J. Antimicrob. Chemother. 74, 1469-1472. doi: 10.1093/jac/ dkz097

Sharma, D., Garg, A., Kumar, M., and Khan, A. U. (2019a). Proteome profiling of carbapenem-resistant K. pneumoniae clinical isolate (NDM-4): Exploring the mechanism of resistance and potential drug targets. J. Proteomics. 200, 102-110. doi: 10.1016/j.jprot.2019.04.003

Sharma, D., Garg, A., Kumar, M., Rashid, F., and Khan, A. U. (2019b). Downregulation of flagellar, fimbriae, and Pili proteins in carbapenem-resistant Klebsiella pneumoniae (NDM-4) clinical isolates: a novel linkage to drug resistance. Front. Microbiol. 10:2865. doi: 10.3389/fmicb.2019.02865

Sharma, D., Misba, L., and Khan, A. U. (2019c). Antibiotics versus biofilm: an emerging battleground in microbial communities. Antimicrob. Resist. Infect. Control 8:76. doi: 10.1186/s13756-019-0533-3

Siala, W., Rodriguez-Villalobos, H., Fernandes, P., Tulkens, P. M., and Van Bambeke, F. (2018). Activities of combinations of antistaphylococcal antibiotics with fusidic acid against staphylococcal biofilms inin vitro static and dynamic models. Antimicrob. Agents Chemother. 62, e518-e598. doi: 10.1128/AAC. 00598-18

Smith, T. T., Tamma, P. D., Do, T. B., Dzintars, K. E., Zhao, Y., Cosgrove, S. E., et al. (2018). Prolonged linezolid use is associated with the development of linezolid-resistant Enterococcus faecium. Diagn. Micr. Infec. Dis. 91, 161-163. doi: 10.1016/j.diagmicrobio.2018.01.027

Strukova, E. N., Portnoy, Y. A., Romanov, A. V., Edelstein, M. V., Zinner, S. H., Firsov, A. A. et al. (2016). Searching for the optimal predictor of ciprofloxacin resistance in Klebsiella pneumoniae by using in vitro dynamic models. Antimicrob. Agents Chemother. 60, 1208-1215. doi: 10.1128/AAC. 02334-15

Suzuki, S., Horinouchi, T., and Furusawa, C. (2014). Prediction of antibiotic resistance by gene expression profiles. Nat. Commun. 5:5792. doi: 10.1038/ ncomms6792

Torres, C., Alonso, C. A., Ruiz-Ripa, L., León-Sampedro, R., Del Campo, R., Coque, T. M. et al. (2018). Antimicrobial resistance in Enterococcus spp. of animal origin. Microbio. Spectr. 6:30051804. doi: 10.1128/microbiolspec.arba0032-2018
Tsuji, Y., Holford, N. H. G., Kasai, H., Ogami, C., Heo, Y., Higashi, Y., et al. (2017). Population pharmacokinetics and pharmacodynamics of linezolid-induced thrombocytopenia in hospitalized patients. Brit. J. Clin. Pharmaco. 83, 17581772. doi: 10.1111/bcp.13262

Tyers, M., and Wright, G. D. (2019). Drug combinations: a strategy to extend the life of antibiotics in the 21st century. Nat. Rev. Microbio. 17, 141-155. doi: 10.1038/s41579-018-0141-x

Vassilara, F., Galani, I., Souli, M., Papanikolaou, K., Giamarellou, H., Papadopoulos, A. et al. (2017). Mechanisms responsible for imipenem resistance among Pseudomonas aeruginosa clinical isolates exposed to imipenem concentrations within the mutant selection window. Diagn. Micr. Infec. Dis. 88, 276-281. doi: 10.1016/j.diagmicrobio.2017.04.005

Vranakis, I., Goniotakis, I., Psaroulaki, A., Sandalakis, V., Tselentis, Y., Gevaert, K., et al. (2014). Proteome studies of bacterial antibiotic resistance mechanisms. J. Proteomics 97, 88-99. doi: 10.1016/j.jprot.2013.10.027

Wang, L., He, Y., Xia, Y., Wang, H., and Liang, S. (2014). Investigation of mechanism and molecular epidemiology of linezolid-resistant Enterococcus faecalis in China. Infect. Genet. Evol. 26, 14-19. doi: 10.1016/j.meegid.2014. 05.001

Wang, Y., Lv, Y., Cai, J., Schwarz, S., Cui, L., Hu, Z., et al. (2015). A novel gene, optrA, that confers transferable resistance to oxazolidinones and phenicols and its presence in Enterococcus faecalis and of human and animal origin. J. Antimicrob. Chemotehr. 70, 2182-2190. doi: 10.1093/jac/dkv116

Wentao, N., Guobao, L., Jin, Z., Junchang, C., Rui, W., Zhancheng, G., et al. (2018). In vitro activity of minocycline combined with aminoglycosides against Klebsiella pneumoniae carbapenemase-producing K. pneumoniae. J. Antibiot. 71, 506-513. doi: 10.1038/s41429-017-0024-9

Xu, S., Fu, Z., Zhou, Y., Liu, Y., Xu, X., and Wang, M. (2017). Mutations of the transporter proteins GlpT and $U h p T$ confer fosfomycin resistance in Staphylococcus aureus. Front. Microbiol. 8:914. doi: 10.3389/fmicb.2017.00914

Xu, X., Xu, L., Yuan, G., Wang, Y., Qu, Y., and Zhou, M. (2018). Synergistic combination of two antimicrobial agents closing each other's mutant selection windows to prevent antimicrobial resistance. Sci. Rep. 8:7237. doi: 10.1038/ s41598-018-25714-Z

Yan, J., Xia, Y., Yang, M., Zou, J., Chen, Y., Zhang, D., et al. (2018). Quantitative proteomics analysis of membrane proteins in Enterococcus faecalis with lowlevel linezolid-resistance. Front. Microbiol. 9:1698. doi: 10.3389/fmicb.2018. 01698

Zahedi Bialvaei, A., Rahbar, M., Yousefi, M., Asgharzadeh, M., and SamadiKafil, H. (2017). Linezolid: a promising option in the treatment of Gram-positives. J. Antimicrob. Chemotehr. 72, 354-364. doi: 10.1093/jac/dkw450

Zhang, X., Bi, W., Chen, L., and Zhang, Y. (2019). Molecular mechanisms and epidemiology of fosfomycin resistance in Enterococci isolated from patients at a teaching hospital in China from 2013 to 2016. J. Global Antimicrob. Resis. 20, 191-196. doi: 10.1016/j.jgar.2019.08.006

Zinner, S. H., Gilbert, D., Lubenko, I. Y., Greer, K., and Firsov, A. A. (2008). Selection of linezolid-resistant Enterococcus faecium in an in vitro dynamic model: protective effect of doxycycline. J. Antimicrob. Chemotehr. 61, 629-635. doi: 10.1093/jac/dkm542

Zinner, M. V. G. E., Yury, A., and Portnoy, A. A. F. (2018). Predicting antibiotic combination effects on the selection of resistant Staphylococcus aureus: in vitro model studies with linezolid and gentamicin. Int. J. Antimicrob. Agents 52, 854-860. doi: 10.1016/j.ijantimicag.2018.09.005

Zou, J., and Xia, Y. (2020). Molecular characteristics and risk factors associated with linezolid-resistant Enterococcus faecalis infection in southwest china. J. Global Antimicrob. Resis. 22, 504-510. doi: 10.1016/j.jgar.2020.03.027

Conflict of Interest: The authors declare that the research was conducted in the absence of any commercial or financial relationships that could be construed as a potential conflict of interest.

Copyright (C) 2021 Jiang, Xie, Chen, Liu, Wang, Mao, Li and Huang. This is an open-access article distributed under the terms of the Creative Commons Attribution License (CC BY). The use, distribution or reproduction in other forums is permitted, provided the original author(s) and the copyright owner(s) are credited and that the original publication in this journal is cited, in accordance with accepted academic practice. No use, distribution or reproduction is permitted which does not comply with these terms. 